\title{
The Causes and Countermeasures of School Bullying in Primary and Middle Schools: From the Perspective of Frustration Aggression Theory
}

\author{
Zhao Kang ${ }^{1, a,{ }^{*} \quad \text { Cai Chen }}{ }^{2, b}$ and Jinyi Shu ${ }^{3, c}$
}

${ }^{1}$ School of Education, Research Center for Mental Health Education, China West Normal University, Nanchong, Sichuan, China

2 School of Foreign Languages, China West Normal University, Nanchong, Sichuan,China

${ }^{3}$ Nanchong No. Eleven Middle School, Nanchong, Sichuan, China

akangzhao168@126.com, b644593460@qq.com,c963982763@qq.com

${ }^{*}$ Corresponding author

Keywords: Primary and middle schools; School bullying; Frustration-aggression theory.

\begin{abstract}
At present, bullying incident in primary and middle schools has aroused widespread concern from people in all walks of life. It is important to analyze the causes and countermeasures of bullying behavior in primary and middle schools. The frustration aggression theory can provide a new perspective for the study of bullying behavior in primary and middle schools. Combining the frustration aggression theory with scientific interpretation of the causes of bullying behavior in primary and middle schools, this paper proposes a good way to effectively deal with the bullying problems in primary and middle schools. Advocating the practice of education should pay attention to the early education of primary and middle school students, guide students to correctly treat all kinds of setbacks and emergencies in daily life, improve students' ability of self-protection and control, scientifically grasp the punishment intensity of primary and middle school students and avoid unlawful infringement and serious consequences caused by bad stimuluses such as verbal violence and injuries.
\end{abstract}

\author{
中小学校园欺凌问题成因及对策：“挫折-攻击”理论视角 \\ 康钊 $1, \mathrm{a},{ }^{*}$ ，陈才 $2, \mathrm{~b}$ ，舒晋一 $3, \mathrm{c}$ \\ 1 西华师范大学教育学院 心理健康教育研究中心，南充，四川，中国 \\ 2 西华师范大学外国语学院，南充，四川，中国 \\ 3 四川省南充市第十一中学，南充，四川，中国 \\ akangzhao168@126.com, b644593460@qq.com, 9963982763@qq.com \\ “通讯作者
}

关键词: 中小学; 校园欺凌; “挫折-攻击” 理论

中文摘要. 当前, 中小学校园欺凌事件引起社会各界人士的广泛关注, 认真分析中小学校园 欺凌行为成因及对策成为学校教育的重要工作。“挫折-攻击” 理论能为中小学校园欺凌行为 的研究提供较新的视角。结合 “挫折一攻击” 理论对中小学校园欺凌行为成因的科学解读, 提出有效应对中小学校园欺凌问题的良方。主张教育实践中应当重视对中小学生个体的早期 教育, 引导学生正确对待日常生活中遭遇的各种挫折和突发事件, 提高学生的自我保护与控 
制能力, 科学把握对中小学生的惩戒力度, 避免言语暴力伤害等不良刺激诱因对中小学生造 成不法侵害和严重后果。

\section{1. 引言}

近年来，我国中小学校园欺凌事件频繁发生，严重影响着青少年学生的健康成长，并对 学校正常的教育教学秩序产生极大干扰, 这一问题逐渐受到社会各界人士的积极关注。国务 院教育督导委员会办公室于 2016 年 4 月专门印发了《关于开展校园欺凌专项治理的通知》, 要求各地教育行政部门和相关机构要针对校园欺凌问题开展专项治理工作，这可看作是从国 家层面提出的对校园欺凌进行治理的总体要求, 由此可见这一问题的重要性和紧迫性, 我们 绝对不能等闲视之。张文新 (2002) 通过对 9235 名中小学生进行的校园欺凌问题专项调查发 现, 小学阶段学生群体中曾经遭受欺凌者比例高达 $22.2 \%$, 对他人曾经实施过欺凌行为者占 比为 $6.2 \%$; 初中阶段学生曾遭受他人欺凌和对他人实施欺凌行为者所占比例分别为 $12.4 \%$ 和 $2.6 \%$; 从总体数据上看有近两成的中小学生曾经卷入校园欺凌问题 $[1]$ 。而且, 相比个体遭受 肉体的创伤来讲, 受欺凌学生心灵的伤害程度可能更深、持续时间可能更久, 严重者可能因 此而表现出抑郁惊恐、暴力攻击、肢体自残甚至自杀轻生等极端行为。认真分析中小学校园 欺凌行为的成因与对策成为当前学校教育的重要工作, “挫折-攻击”理论能为科学理解和诠释 中小学校园欺凌事件提供全新的视角。

\section{2. 中小学校园欺凌行为的概念理解及特征分析}

\section{1 中小学校园欺凌行为的概念理解}

国内学者常将英文 “Bullying”一词译作 “欺凌”，也有人将其译为 “霸凌” 或 “欺负” 等，含有 “恃强凌弱” 或 “仗势欺人”之意。何谓校园欺凌？可以说，时至今日，国内学术 界和司法界对于其概念还尚未形成统一的认识, 部分学者将其与 “校园暴力” 概念等同起来。 但随着国家机关正式文件下达，使用 “校园欺凌”一词者相对较多并逐渐趋同。有学者认为 校园欺凌是指以校园作为背景而发生的一方对另一方进行的蓄意攻击，具体包括身体和言语 以及关系和网络等各个层面实施的攻击行为 [2]。胡春光 (2017) 认为校园欺凌是指学校环境中 发生的学生群体或个别学生故意重复地对那些可能不会对其实施报复行为的受害学生施以长 期性的身体或心理伤害的攻击形式 [3]。俞凌云、马早明 (2018) 将其界定为在校人员借助某种 权力长期压迫其他在校人员, 造成他人生理、心理伤害或干扰正常教学秩序的行为 $[4]$ 。任海 涛（2017）则认为应当从狭义方面对校园欺凌进行界定，并与校园暴力明确区分，他提出的 概念涉及发生空间、欺凌者与受害人、欺凌特征、地位、影响及表现形式等内容 [5]。尽管不 同学者对其概念界定不一，但大都认为校园欺凌与学校师生高度相关，主观上有实施欺凌的 恶意, 客观上对受欺凌者造成伤害, 通常是以相对隐蔽和持久的攻击行为表现出来, 一般较 难为人发现。而且实施欺凌行为者与受欺凌者之间存在着较为明显的力量差异, 欺凌者常常 以对受欺凌者实施身心方面的伤害为直接目的, 意图较为明确, 受欺凌者可能较长时期处于 一种无力抵抗而被迫接受欺凌者一切攻击行为的状态。

\section{2 当前中小学校园欺凌行为的特征举隅}

当前校园欺凌事件在实践中的表现形式逐渐呈现多样化特点，具体而言可将其分为身体 欺凌、言语欺凌和关系欺凌等方式，既有直接面对面或身体接触的外显欺凌形式，也有借助 网络媒体进行的相对隐蔽的欺凌方式。综观针对校园欺凌问题进行的诸多研究，表明校园欺 凌大多具有形式多样化、作案团伙化、行为隐蔽性、过程持续性及手段残忍性等特征。段威

（2018）认为当前校园欺凌现象频发与社会和家庭结构的变迁有着紧密的联系，并表现出欺 凌手段的多样性、传播的高效性、危害结果的持续性和治理难度不断增加等特性 [6]。认真分 
析近年来发生在全国各地的中小学校园欺凌事件，结合国内外众多学者对这一问题的深入研 究, 我们可以看出当前中小学校园欺凌行为呈现出诸多新的特征。首先, 中小学校园欺凌行 为的发生地由校园内部逐渐转移至校外场所或借助网络媒体实施侵害。相对来说, 因当前校 园欺凌行为转向校园邻近区域、学生上学和放学沿途、较为偏僻或灯光昏暗的地方等隐秘区 域而更难发现, 而借助网络进行的欺凌行为对学生产生的影响和伤害可能更大。其次, 校园 欺凌行为由原来的团伙作案逐渐转向个体单独行动。许多欺凌者在实施欺凌行为时多采取单 打独斗的方式进行，这样更不容易被外界知晓而露馅。第三，由于城区学校监管网络体系的 高度发达, 学校教育防控力度加强, 学生的自我防范和反欺凌意识大大提升, 中小学校园欺 凌行为高发地由原来的城镇逐渐转向城郊结合部或农村学校。第四, 欺凌行为与欺凌者自身 的性格特点、交往能力、学习能力、身体条件和家庭环境有关, 校园欺凌行为实施者和受欺 凌者的两极特征表现较为鲜明。欺凌他人者多表现为霸道狂妄、社会朋友较多、学业成绩不 良、体格健壮、家境较为殷实富有，而被欺凌者常表现为胆小怕事、性格内向、自卑退缩等 特征, 两者之间反差特别明显。此外, 虽然许多中小学生在遭受校园欺凌时能够积极寻求帮 助来解决问题, 但由于欺凌者过于强势或者学校师生和家长应对不力, 或委曲求全而逆来顺 受, 由此造成部分受欺凌者对校园欺凌问题的消极应对。这在一定程度上增加了学校解决校 园欺凌问题的难度，对此我们必须要有清醒的认识并积极应对。

\section{3. “挫折-攻击”理论内涵及其对中小学校园欺凌事件的成因解读}

许多学者在针对校园欺凌问题进行研究时都有这样一种认识，即认为中小学校园欺凌行 为就是中小学生遭遇挫折之后的日常攻击行为的极端体现。综观目前对校园欺凌问题的理论 解释多以攻击理论为基础, 我们认为 “挫折-攻击” 理论的确能够为解释中小学校园欺凌行为 提供科学的理论视角。

“挫折 - 攻击”理论（ frustration-aggression theory）也称作 “挫折 - 攻击”假说 （frustration-aggression hypothesis），1939 年由美国耶鲁大学著名心理学家 Dollard 联合其同 事 Miller 等首次提出。该理论认为当个体的某些需要得不到满足时可能诱发个体因此体验到 强烈的挫折感，进而个体通过攻击行为来缓解或掩盖自身所遭遇的挫折感。可以说，那些经 历过挫折的个体常常会表现出攻击行为, 那些表现出攻击行为的个体常常遭受过挫折。“挫折 -攻击”理论将“个体的攻击行为始终是个体曾经遭受挫折的结果”作为其理论假设, 认为个体 的挫折经历是出现攻击行为的前提条件，把个体的攻击行为看成是对挫折的自动反应 [8]。个 体所遭遇的挫折往往总会导致某种形式的指向所有阻碍达成其目标的个体或其他对象的攻击 行为, 而且相对严重的挫折可能还会促成个体产生敌意、威胁、报复等不良心理乃至出现暴 力犯罪行为[9]。然而, 在实践中人们发现攻击行为也并非完全由挫折而引起, 如中小学生在 他人的威胁恐吓或唆使利诱等情境下也可能引发攻击行为。因此, 用“挫折一攻击”假说来解 释个体缘何出现攻击行为很快就暴露其理论缺陷与不足之处 [10]。特别是随着时间的推移和 研究工作的不断深入，人们逐渐意识到早期提出的“挫折-攻击”理论假设及观点过于简单和概 括, 而且对许多涉及挫折和攻击行为的现象都无法作出较为满意的解释, 因为生活实践中的 确有很多人在遭受挫折以后并没有发生任何攻击行为 [11]。为了进一步科学解释这些现象和 完善相关理论研究的不足, Miller 等(1941)对他们的研究立场和观点重新作出调整, 提出个体 在遭遇挫折时可能会出现借助间接形式来加以表达的攻击行为。这种间接攻击行为的表现形 式复杂多样, 最明显和直接的表现是将其攻击行为迁移到另外一个新的目标上面。如个体将 自身在工作中遭遇的挫折产生的不良情绪发泄到配偶或他人身上，或者个体不能直接与自己 的上司发生正面冲突，但却可能通过在工作中故意施计作梗或散布谣言来加以报复。从这些 意义上说, 个体遭受挫折后可能会表现出攻击行为, 但不一定非得采用明显或外显的攻击方 式。

其实, 在这之前的许多学者也对个体的攻击行为这一问题作过深入研究。如奥地利著名 
精神分析学家弗洛伊德 (Freud) 提出的本能理论, 认为个体的一切活动都来源于天生的性本 能, 个体之所以出现攻击外部事物或者他人的行为是因为个体自身被长期压抑的性冲动得到 充分发泄和释放的结果。奥地利习性学家、诺贝尔生物学奖获得者劳伦兹 (Lorenz) 和现代 的一些研究也持有类似的观点, 认为个体自身原本可能具备一定的先天攻击倾向, 即个体的 攻击性在某种程度上深受遗传因素的影响而与外界刺激诱因并没有太多关联。但在教育与生 活实践中我们很难对本能理论进行评价, 因为对个体身上是否具有攻击本能这一问题极难证 实或证伪, 其理论局限性自然呈现。美国著名社会心理学家班杜拉认为个体在社会学习过程 中不断通过模仿强化习得侵犯或攻击行为, 当个体遭遇挫折时就可能用侵犯或攻击行为来求 得新的平衡状态。这一理论虽然未能对攻击行为发生的内部机制作出明确界定, 但也能提示 我们应当重视科学对待中小学生日常生活中遭遇的各种挫折并为其提供良好的行为典范以减 少攻击行为的发生。上个世纪 60 年代伯科维茨（Leonard Berkowitz）也充分意识到 “挫折攻击” 理论的局限性并在原来的基础上提出经过修正的理论模型。他认为攻击行为不是个体 天生的本能, 也不是挫折的唯一行为结果, 个体攻击行为的出现依赖于个体对所遭受挫折的 认同和厌恶程度。个体所遭遇的挫折本身并不会直接导致个体对外发生攻击性行为, 而只是 在某种程度上为其创造了激活唤醒或准备状态。个体攻击行为是否发生主要取决于外界线索 引导, 若外界线索将个体行为引向积极方向则产生积极行为, 反之则可能产生消极行为。个 体欺凌行为的产生与欺凌者对外界线索的解读有关, 而引发欺凌行为的原因具体包括两个: 个体内在的准备度即主体决定是否参与欺凌行为、个体对外界环境的解读即主体决定产生积 极行为或消极行为进而决定欺凌行为是否发生。这个修正的理论模型能够对实践中部分挫折 不会导致个体出现攻击行为, 以及个体不同的看法会增加或减少攻击行为的可能性作出较为 科学的解释。研究同时还发现, 如果个体因他人原因而受挫, 则个体实施攻击行为的强弱程 度可能会依据个体对他人行为的主观故意状况来作出判断。由此，伯科维茨将 “情绪唤醒” 这一中介变量加入 “挫折一攻击” 理论模型之中, 使得该理论能够更加科学合理地解释人类的 攻击行为, 其理论体系也逐渐为人们所接受。然而, 实践中我们稍加考虑就会发现个体攻击 行为的出现可能受制于除挫折以外的其他多种因素, 诸如个体身上出现的某些工具性攻击行 为。相关理论研究没有充分考虑个体的主观认知对攻击行为的调控作用, 也未对情绪唤醒机 制做出明确的阐释。因为要真正理解个体的攻击行为意图, 必须充分考虑个体的心理活动整 体特点并由此作出全面科学的认识。

应当说, 发生在中小学校园中的许多欺凌事件, 我们的确可以用 “挫折-攻击” 理论对 其进行科学解释。我们可以看出, 中小学生容易因缺少家长或老师的保护而被欺凌, 或者通 过欺凌他人来彰显自己的独立与力量。正是由于许多学习困难或有品行障碍的学生在日常学 习与生活没有得到老师或其他同学的尊重和理解, 容易经常被老师和其他同学误解或怀疑, 由此产生较为强烈的挫折感和不悦的情绪体验, 而这些个体一旦对外界不利的线索进行错误 解读, 同时个体自己又不得不努力去缓解或掩盖自身遭受的挫折感, 则极大可能由此产生消 极的应对方式或不良行为，直接导致其产生攻击行为或成为欺凌的对象。

\section{4. 中小学校园欺凌事件应对策略探讨一一基于“挫折-攻击”理论视角}

\section{1 重视对中小学生个体的早期教育是有效预防校园欺凌事件发生的第一步}

“挫折一攻击”理论提示我们，倘若能在教育实践中多为学生提供正向的引导线索，必然 在很大程度上降低或减少校园欺凌事件的发生。因此, 学校教师和家长重视对中小学生个体 进行正面的早期引导教育很有必要。当前, 许多学生家长对家庭早期教育在预防校园欺凌事 件的重要性认知不足, 甚至部分家长长期坚信 “树大自然直”, 认为孩子长大以后一切问题 症状或行为都会自然消失, 所以对孩子疏于管教, 更不注重自身的不良言行举止对孩子潜移 默化地产生负面影响。许多研究表明中小学生个体身上攻击性行为的出现与个体早期的不良 
教育和对某些攻击行为的自动习得有关, 因此, 在个体成长的童年或较早时期应当特别重视 父母或其他相关人员对个体的言传身教和教育引导功能, 避免攻击行为的早期习得。同时, 正因为儿童具备较强的模仿学习能力, 应该尽量对中小学生所接收的诸多传媒信息做出过滤, 以避免中小学生从不良的大众传播媒介中去简单模仿或无意之中习得攻击行为。我们应当加 强对中小学生的教育引导, 将良好的社会教育内容尽可能较早地融入儿童的生活与学习之中, 增强个体的道德认知能力, 促进其社会化进程的顺利实现和完善其健康人格, 提升个体的社 会化水平。

\section{2 引导中小学生科学认知校园欺凌现象, 正确对待日常生活中遭遇的各种挫折}

中小学校应当重视采用科学的认知训练方法对中小学生的校园欺凌行为做出干预, 改善 其不良认知以减少欺凌行为的发生概率。部分学生对欺凌行为存在认知偏差, 如将欺凌行为 视作 “引起关注” 或是 “树立威风” 的手段, 认为让别人害怕自己则自己很有面子; 而被欺 凌者不愿意将此事告知他人的重要原因就是担心 “别人知道后会看不起自己” [7]。这些不良 认知严重扭曲中小学生的价值观念, 并且极易助长校园欺凌现象的滋生和蔓延, 学校管理者 和广大教师必须高度重视并强化引导工作。刘丽娜（1998）认为个体对挫折的来源、性质、 强度等情境的认知和理解在很大程度上影响个体侵犯性欲望和情感的变化, 进而影响个体的 行为反应方式 [12]。但同时也应当看到, 对挫折情境的不同理解虽然可能影响到个体的行为 反应方式, 但这并不能决定个体必然出现这种行为反应。生活中我们可以见到有些人遭遇挫 折后情绪极度低落, 甚至异常愤怒或难于自制, 但他个人可能并没有出现各种攻击行为反应。 而另外一些人无心侵犯他人却在无意之中招惹了他人。个体的情感受制于个体的道德认识、 社会情感与态度、法律意识等因素, 事实上只有当一个人真正面对问题或者接近侵犯性反应 时, 才有可能产生较大的个人焦虑。个体在社会化进程中, 如能较早内化社会行为规范, 合 理调控自己的情绪情感, 并由此形成基本的法律意识, 这样当个体遭遇到可能引发攻击行为 的情境时也能泰然处之。

\section{3 科学把握对中小学生的惩戒力度, 防止过度惩罚导致中小学生出现攻击行为}

已有的研究表明：个体如若遭受过度惩罚则可能因过度愤怒而出现攻击或欺凌行为予以 报复性应对，有时也可能因为获取利益或好处而甘受惩罚。前苏联著名教育家苏霍姆林斯基 曾明确告诫我们：惩罚是一种不无危险的教育手段。因为惩罚受制于个体的认识、情感与意 志等因素的影响, 惩罚必须科学适度, 更重要的是要具有一定的威慑性和教育性, 才能真正 发挥其教育功效。特别是中小学生善于模仿别人的行为并从中获取好处, 如同个体在家中为 了避免父母的惩罚可能不再从事攻击性行为, 但父母对子女的不当教育方式特别是过度惩罚 可能会在个体心中留下深刻的印象与痕迹, 当这些孩子遭遇类似情境时, 他们也可能用同样 的方式去对待他人而实施攻击行为。认为那些经常实施攻击行为的人, 多半是在儿童时期经 常受到父母、兄长或者其他有关的人的严厉惩罚 [12]。同时, 我们更应当看到部分学生可能 因为遭受不当惩罚而自暴自弃、破罐破摔。因此, 我们在日常教育中应当注意科学把握对中 小学生的惩戒力度, 帮助他们正确面对可能遭遇的各种问题, 重视培养他们良好的社会责任 感和使命感，防止对他们的过度惩罚而出现攻击行为。

\section{4 引导中小学生正确处理各种突发事件和不良刺激, 提高中小学生的自我保护与控制能力}

特别应当注意的是，当前不良网络媒体信息泛滥造成暴力文化与黑恶势力等消极内容严 重扭曲青少年的价值观和人生观, 某些语言、情境或事件可能成为一种不良刺激诱因对处于 正在成长发展的中小学生造成极大的影响和伤害, 并由此引发校园欺凌或攻击行为的产生。 事实上, 当前中小学校园欺凌事件中言语欺凌占比较高, 这提示我们应当特别关注中小学生 的生存环境, 避免言语暴力伤害等外界不良刺激诱因对中小学生造成的不法侵害, 全力建设 良好的校园环境和网络传播氛围, 为中小学生的健康成长保驾护航。在教育教学中我们应当 
重视对中小学生进行心理健康教育工作, 科学推广和全面普及心理健康知识, 提升中小学生 的心理素养水平, 学会情绪的自我调节与控制, 尽量减少卷入日常生活中可能诱发不良情绪 的各种情境, 提高学生的自我控制能力。要科学引导中小学生正确处理各种突发事件和外界 不良刺激, 善于从学生的日常生活中发现问题并及时处理, 特别应当及早发现存在异常情绪 或行为表现的学生并及时干预。各级教育主管部门和学校要加强对中小学生的法治教育并将 其有效融入学校课程体系, 增强中小学生的法治意识, 提高中小学生遵纪守法的自觉性, 开 展系列活动强化中小学生的自我保护意识和提升其自我防范能力, 逐渐学会并养成利用法律 武器维护自身合法权益的良好习惯。

\section{致谢}

本文为四川省教育厅 [川教函 (2017) 336号]教育科研重点课题: 中小学校园欺凌行为及干 预策略研究; 四川省哲学社会科学重点研究基地一一四川省教育发展研究中心科研项目: 中 小学校园欺凌问题研究（CJF17028）项目研究成果之一。

\section{References}

[1] Zhang Wenxin, Prevalence and Major Characteristics of Bullying/Victimization among Primary and Junior Middle School Children, Acta Psychologica Sinica, Vol. 34, No.4, pp. 387-394, 2002.

[2] Sun Shijin, Shi Zeyi, The Psychological Factors and Treatment of School Bullying, Journal of East China Normal University (Educational Sciences), Vol. 35, No.2, pp. 51-56, 2017.

[3] Hu Chunguang, School Bullying Behavior: Meaning, Cause and Prevention Strategy, Educational Research and Experiments, No.1, pp. 73-79, 2017.

[4] Yu Lingyun, Ma Zaoming, "Campus Bullying": Connotation, Limits of Application and Redefinition, Educational Development Studies, No.18, pp. 26-32, 2018.

[5] Ren Haitao, The Definition of School Bullying and its Legal Liability, Journal of East China Normal University (Educational Sciences), No.2, pp. 43-50, 2017.

[6] Duan Wei, Causes and Prevention of School Bullying--- Dialogue between Law and Sociology, Juvenile Delinquency, No.2, pp. 22-27, 2018.

[7] Xue Lingling, Wang Weihong, Feng Xiao, Campus Bullying in Multiple Prevention and Control: Based on the Investigation and Analysis of the Situation of Campus Bullying in C City Primary and Secondary Schools, Educational Scientific Research, No.3, pp. 24-29, 2018.

[8] Liu Yilin, Zeng Lianping, Yang Zhongyu, Huang Man, Review of Research on Aggression among Juveniles, Journal of Henan Police College, Vol. 23, No.4, pp. 51-56, 2014.

[9] Li Anheng, The Exploration of John Barton's Criminal Cause Based on the Frustration-Aggression Hypothesis, Journal of Shaoguan University (Social Science), Vol. 35, No.7, pp. 24-27, 2014.

[10] He Xianyou, Psychological Explanation of Human Aggression, Journal of Xiangtan Teachers College, Vol. 15, No.4, pp. 72-74, 1994.

[11] Song Shujuan, Summary of Theoretical Research on Attack Behavior, Science of Social Psychology, Vol. 17, No.4, pp. 23-26, 2002.

[12] Liu Lina, On Frustration and Infringement, Science of Social Psychology, No.1, pp. 52-56, 1998. 